Document downloaded from:

http://hdl.handle.net/10251/73090

This paper must be cited as:

Fuster García, E.; Bresó Guardado, A.; Martínez Miranda, JC.; Rosell-Ferrer, J.; Matheson, C.; García Gómez, JM. (2015). Fusing actigraphy signals for outpatient monitoring. Information Fusion. 23:69-80. doi:10.1016/j.inffus.2014.08.003.

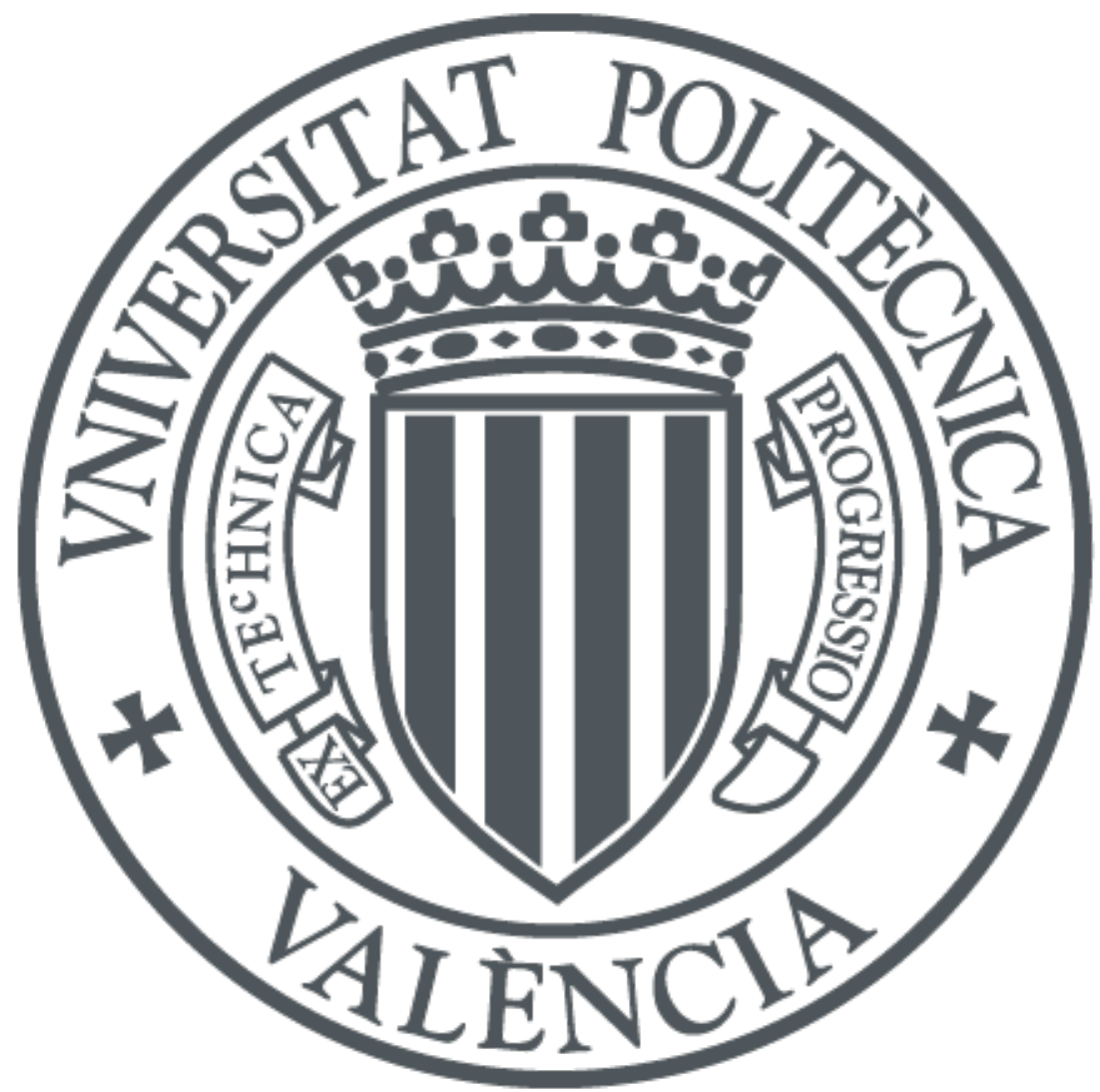

The final publication is available at

http://dx.doi. org/10.1016/j.inffus.2014.08.003

Copyright Elsevier

Additional Information 


\title{
Fusing actigraphy signals for outpatient monitoring
}

\author{
Elies Fuster-Garcia ${ }^{\mathrm{a}, *}$, Adrián Bresób, Juan Martínez-Miranda ${ }^{\mathrm{b}}$, Javier \\ Rosell $^{\mathrm{c}}$, Colin Matheson ${ }^{\mathrm{d}}$, Juan M. García-Gómez ${ }^{\mathrm{b}}$ \\ ${ }^{a}$ Veratech for Health, S.L., València, Spain \\ ${ }^{b}$ Instituto de Aplicaciones de las Tecnologías de la Información y de las Comunicaciones \\ Avanzadas (ITACA), Universitat Politècnica de València, València, Spain \\ ${ }^{c}$ Departament d'Enginyeria Electrònica, Universitat Politècnica de Catalunya, \\ Barcelona, Spain \\ ${ }^{d}$ Institute for Language, Cognition, and Computation, University of Edinburgh, \\ Edinburgh, Scotland, UK
}

\begin{abstract}
Actigraphy devices have been successfully used as effective tools in the treatment of diseases such as sleep disorders or major depression. Although several efforts have been made in recent years to develop smaller and more portable devices, the features necessary for the continuous monitoring of outpatients require a less intrusive, obstructive and stigmatizing acquisition system. A useful strategy to overcome these limitations is based on adapting the monitoring system to the patient lifestyle and behavior by providing sets of different sensors that can be worn simultaneously or alternatively. This strategy offers to the patient the option of using one device or other according to his/her particular preferences. However this strategy requires a robust multi-sensor fusion methodology capable of taking maximum profit from all of the recorded information. With this aim, this study proposes two actigraphy fusion models including centralized and distributed architectures based on artificial neural networks. These novel fusion methods were tested both on synthetic datasets and real datasets, providing a parametric characterization of the models' behavior, and yielding results based on real case applications. The results obtained using both proposed fusion models exhibit good performance in terms of robustness to signal degradation, as well as a
\end{abstract}

${ }^{*}$ Corresponding author: Elies Fuster-Garcia, Veratech for Health S.L., Gran Via Ferran El Catlic 5, València, Spain, Telf.: 627015023

Email address: efuster@veratech.es (Elies Fuster-Garcia) 
good behavior in terms of the dependence of signal quality on the number of signals fused. The distributed and centralized fusion methods reduce the mean averaged error of the original signals to $44 \%$ and $46 \%$ respectively when using simulated datasets. The proposed methods may therefore facilitate a less intrusive and more dependable way of acquiring valuable monitoring information from outpatients.

Keywords:

actigraphy, multi-sensor fusion, outpatient monitoring, major depression, artificial neural networks

\section{Introduction}

In recent years there has been growing interest in the development of computer based health systems to facilitate prevention, early diagnosis, and treatment through the continuous monitoring of patients outside clinical institutions. These systems are able to provide immediate and personalized health services to individuals regardless of location, facilitating normalization of the patient's lifestyle during treatment thereby enhancing their life quality.

One of the biggest challenges in the development of these computer based systems is the monitoring of the mental, physiological, and social signals from the patient without influencing or changing the patient's daily life activities. Due to advances in wireless technologies and wearable electronics, today it is possible to integrate sensors in small and discrete devices, allowing long time non-invasive studies of free-living patient activity [1]. Nevertheless, greater efforts are still required to make these devices as unobtrusive as possible for the target users and those around them.

In order to improve these properties, a multi-sensor based strategy consisting of the use of different devices to monitor the same activity (e.g. wrist watch, smartphones, or undermattress actigraphs) can be used. This strategy gives more flexibility to the patient, allowing the use of one device or another, thus adapting the system to the individual patient's lifestyle and behavior. This methodology minimizes the user's responsibility for the operation of the system, making the system more robust to individual sensor data loss, and making the monitoring system more transparent to the user. 
Outpatient monitoring using a multi-sensor based strategy requires a data fusion model capable of taking maximum profit from all of the generated information, which in most cases is redundant, with non-linear dependencies, long periods of missing data, and different sensitivity levels. In this sense, this study proposes two novel multi-sensor fusion methodologies at the raw level of actigraphy signals. The main goal of the fusion methodology presented here is to obtain a single fused activity signal that improves the information contained in each single original signal, avoiding common artifacts such as noise, missing data, or spurious data, and complementing the loss of sensitivity of some of the sensors used. That is, to design a low signal abstraction level multi-sensor fusion method (data in-data out) for actigraphy signals $[2,3]$. In recent years different multi-sensor data fusion techniques have been presented in the literature $[4,5,6]$. These fusion techniques were designed to deal with the main challenging problems in multi-sensor fusion [5] such as: data imperfection, outliers, conflicting data, multi-modality data, correlation, alignment, data association, processing framework, operational timing, dynamic phenomena, and dimensionality. In the specific case of fusing actigraphy signals for outpatient monitoring, the main problems to overcome are related to data imperfection (impreciseness and uncertainty in the measurements), outliers (artifacts and missed data) and heterogeneous sensors (different sensors, devices, or event placement in the patient body). Each of these problems was addressed separately in the literature considering different approaches. In the case of imperfect data the main approaches followed were the probabilistic $[7,8]$, the evidential $[9,10,11]$, fuzzy reasoning $[12,13,14]$, possibilistic $[15,16]$, rough set theoretic $[17,18,19]$, hybridization $[20,14]$ and random set theoretic $[21,22]$. In the case of outliers and missing data, the most common approaches are based on sensor validation techniques $[23,24,25]$ and on stochastic adaptive sensor modelling [26]. Finally the approaches followed to solve the problems related to heterogeneous sensors were highly depending on the sensors used and the desired target. Multi-sensor data fusion techniques were successfully applied in the specific problem of fusing actigraphy signals. In [27] the authors proposed the use of a Hidden Markov Model to classify daily activities by combining the data coming from three different accelerometers. In [28] a system for activity recognition using multi-sensor fusion based on a Naïve Bayes classifier was presented achieving a $71 \%-98 \%$ recognition accuracies when using 1-4 sensors. In [29] the authors used a hierarchical classifier for activity recognition that combines a decision three classifier (to select a optimum sensors subset) with a Naïve 
Bayes classifier (to classify the activities), in order to reduce energy consumption of the system while maintaining the recognition accuracy. In [30] the authors presented a multi-sensor based method for classification of daily life activities based on a hierarchical classification algorithm and compare their performance with state-of-the-art algorithms by using a benchmark dataset. In [31] the authors fused the features obtained using a set of actigraphy sensors placed in different parts of the body for activity recognition, comparing the performance of five types of classifiers including ANNs, decision tree classifiers, KNN classifier, the Naïve Bayes classifier, and the support vector machine classifier. Guiry et al. studied in [32] the role that smart devices, including smartphones and smartwatches, can play in identifying activities of daily living. They trained different classifiers including C4.5, CART, Nave Bayes, Multi-Layer Perceptrons and Support Vector Machines using both single and multi-sensor approaches (including not only accelerometer but also gyroscope and magnetometer). They conclude that the fusion of the different signals improve the accuracy of the activity identification. In [33] the authors used a set of smartphones including gyroscope, magnetometer and accelerometer placed in different parts of the body for physical activity recognition. One of the main interesting conclusions obtained is that the combination of the different smartphones used only improved the overall recognition performance when their individual performances were not very high. Finally in $[34,35]$ the authors presented a sensor fusion method for assessing physical activity of human subjects, based on support vector machines by using acceleration and ventilation data measured by a wearable multi-sensor device. Their results show that the fusion approach improved the results of the traditional accelerometer-alone-based methods. The abovementioned methods were successfully applied to fuse different actigraphy signals $[27,28,29,32,33]$ or even to fuse actigraphy signals with other types of sensors $[34,35,32,33]$. However, in most cases the result of these techniques have been to obtain better estimates of energy consumption [35, 34] or to improve activity recognition [27, 28, 29, 33, 32, 30], but not to obtain a more robust fused signal based on a low signal abstraction level approach.

The proposed fusion models are based on the transformation of the input signals to a common representation space where they can be combined linearly. To do this, one sensor is chosen as the reference, and a non-linear regression model is designed to transform the rest of the sensor signals to the representation space of the reference sensor. Once the signals are transformed they are comparable and, as a result, a mixing strategy based on 
a weighted sum can be applied to obtain a final fused signal. This mixing strategy should avoid the missed data periods that may distort the fused signal.

In this work the non-linear regression models are based on Artificial Neural Network (ANN). We have considered the two principal multi-sensor data fusion approaches: The first one is a centralized fusion model, in which all the signals (except the reference one) constitute the inputs to a single ANN trained with the reference signal as output. The second one is a distributed fusion model in which each non-reference signal constitutes the input of a different ANN trained with the reference signal as output.

In order to test the proposed fusion method we focus on a real case application where the system properties of transparency to the user, nonstigmatizing technology, and non-obstruction to lifestyle are mandatory. This is the case of monitoring patients who are recovering from major depression. These patients require constant monitoring to assess their emotional state at all times in order to make recommendations for healthy practices and to prevent relapses. Several studies have shown the importance of motor activity as a relevant behavior pattern for assessing patients with depression [36, 37], and for measuring treatment outcomes in major depression $[38,39,40,41,42]$. Moreover, in recent years different research initiatives has focused their efforts in the development of computer based health systems for following up outpatients and for the automatic prescription of healthy practices. Some examples of these projects are the Help4Mood [43], Monarca [44], ICT4Depression [45], and Optimi [46] EU projects. In these projects different actigraphy devices such as smartphones, wrist watches, key-rings, and even undermatress sensors have been used to monitor patients with major depression.

The evaluation of the fusion methodology has been performed using both synthetic and real datasets. This allows a precise characterization of the behavior of the fusion models in controlled conditions, and also the evaluation of the performance of the fusion models in a real case application. In this work a novel methodology for synthetic actigraphy data simulation has been prepared to perform an exhaustive evaluation. This simulation methodology includes: a pipeline for signal preprocessing, a non-linear pre-processing algorithm based on Functional Data Analysis (FDA), a feature extraction module based on FDA, and a signal modeling step based on Multivariate Kernel Density Estimation (MKDE).

The adequate functioning of the proposed fusion methodologies will con- 
stitute a significant improvement in the monitoring of the physical activity of outpatients, allowing a less invasive means to acquire more data. Moreover these methods will increase the robustness of the acquisition systems, reducing the effect of individual damaged sensors in the final actigraphy signal used for following up the patient.

\section{Actigraphy data fusion models}

Two different actigraphy raw data fusion methods based on centralized and distributed architectures are presented. In both cases the main idea is to take one of the input signals as a reference signal, and then to obtain a transformation model capable of transforming the rest of the input signals to the representation space of the reference signal. The reference signal must be chosen following signal quality criteria. Thus we choose the signal which has the best features in terms of amount of missing data, sensitivity to patient motion, or noise, as a reference signal. Once all the input signals are in the same representation space we are able to combine them linearly. To implement this, there are two main modules in the data fusion methods, the transformation module and the mixing module.

Both methods include transformation modules based on feedforward ANN [47]. The main differences between the methods are related to the combination of the information provided by the different sensors. Hereinafter the $S 1$ signal will denote the actigraphy signal selected to be the reference, and $S 2$, $S 3, \ldots, S N$ will represent the rest of the actigraphy signals obtained from the patient monitoring.

\subsection{Centralized fusion model}

The first data fusion model is based in a centralized fusion architecture (see Figure 1). In this model the non-reference signals are combined and transformed into a single signal $S T$ (in the $S 1$ reference representation space) before being combined with $S 1$. To do this, $S 2, S 3, \ldots, S N$ feed a single ANN to obtain a single transformed signal $S T$ in the $S 1$ reference representation space. Subsequently, the signal $S T$ is normalized and mixed with the refer-

ence signal $S 1$ to obtain the final fused signal $F S$. A detailed description of each step is presented below.

\subsubsection{ANN non-linear regression model}

The ANN used in this model was a feedforward neural network [47]. The architecture of the ANN was selected to be as simple as possible to avoid 
overfitting, while ensuring a precise regression model. To select the number of layers and the number of units per layer we took into account two considerations. The first is the Universal Approximation Theorem [48], which states that a feed-forward network with a single hidden layer containing a finite number of neurons is a universal approximator among continuous functions on compact subsets of $\mathbb{R}^{n}$. The second consideration was the established trial and error rule that states that the number of units in the hidden layer has to be less than twice the size of the input layer [49]. Following these caveats the architecture of the network was defined with an input layer with $N-1$ units, one hidden layer with $2(N-1)$ units, and a single processor in the output layer (see Figure 1). The hidden units used a hyperbolic tangent activation function and the output unit used a linear activation function. The model parameters were obtained from the training data using a back propagation learning algorithm with automatic regularization based on Bayesian interpolation [50]. The ANN was trained using the $S 1$ data as output and the $S 2, .$. , $S N$ data as input, to obtain the non-linear regression model between the $S 2$, ..., $S N$ representation spaces and the reference signal defined by $S 1$.

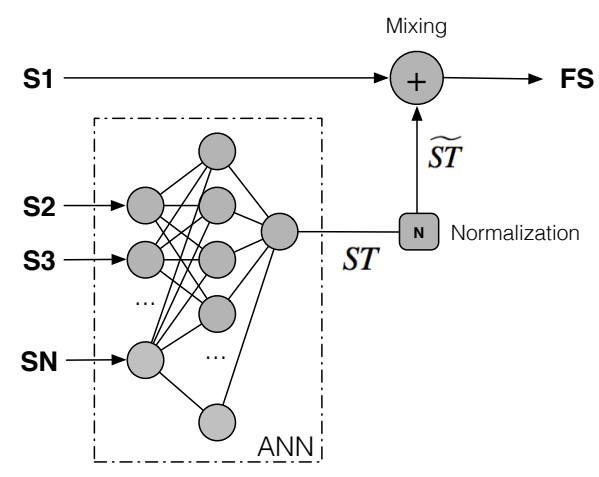

Figure 1: Schema of the centralized fusion model. $S 1$ represents the selected reference signal, $S 2, \ldots, S N$ represent the remaining input signals, $S T$ represents the signal generated by the ANN, $\widetilde{S T}$ is the normalized signal, and $F S$ represents the resulting fused signal.

\subsubsection{Normalization}

When $S 2, S 3, \ldots, S N$ are transformed to $S T$ by the ANN model, an offset could be introduced into the ground level of the signal. This is due to the low sensitivity and the influence of sensor noise. To correct this offset while 
preserving the peak height, a normalization step is applied. In this work the normalization function used is:

$$
\widetilde{S T}(t)=\frac{(S T(t)-\min (S T)) \max (S T * \mathbf{u})}{\max ((S T-\min (S T)) * \mathbf{u})}
$$

where $\mathbf{u} \in\{1\}^{30}$ and $*$ denotes the convolution function.

\subsubsection{Weighting and mixing}

Once $\widetilde{S T}$ is obtained, we linearly mix it with $S 1$ using the expression

$$
F S(t)=W(t) S 1(t)+(1-W(t)) \widetilde{S T}(t)
$$

where $W(t)$ is a weighting coefficient. This coefficient assigns a priori the same contribution to each signal, but avoids mixing missed data. That is, this coefficient drops the contribution of a signal when the algorithm detects that the values of $S 1$ and $S T$ differ, and the signal value is zero. $W(t)$ is defined as

$$
W(t)=\left\{\begin{aligned}
0 & \text { if } S 1(t)=0 \wedge|S 1(t)-\widetilde{S T}(t)|>M+D \\
1 & \text { if } \widetilde{S T}(t)=0 \wedge|S 1(t)-\widetilde{S T}(t)|>M+D \\
1 / N & \text { otherwise }
\end{aligned}\right.
$$

where $M$ is defined as the mean of $|S 1(t)-\widetilde{S T}(t)|, D$ is defined as the standard deviation of $|S 1(t)-\widetilde{S T}(t)|$ and $N$ is equal to the number of sensors fused.

\subsection{Distributed fusion model}

The second data fusion model is based on a distributed fusion architecture (see Figure 2). In this model the non-reference signals $S 2, S 3, \ldots, S N$ are combined and transformed separately into signals $S T 2, S T 3, \ldots, S T N$ (in the $S 1$ reference representation space) before combining them with $S 1$. To do this, each of the non-reference signals feeds a different ANN. Subsequently, the transformed signals are normalized and mixed with the reference signal $S 1$ to obtain the final fused signal $F S$. A detailed description of each step is presented in the following subsections. 


\subsubsection{ANN non-linear regression model}

The distributed model includes as many ANN as the total number of sensors fused minus one (i.g. $N-1$ ). The unique change between the ANN configurations of the centralized and distributed models is the architecture of the ANN (see Figure 2). In this distributed fusion model the ANN has only a single input and therefore we have reduced the number of units of the hidden layers to two (following the considerations mentioned in Section 2.1.1, based on [48] and [49]). The model parameters were obtained from the training data using a back propagation learning algorithm with automatic regularization based on Bayesian interpolation [50]. Each ANN is trained using the $S 1$ data as output and a single sensor data $S i$ as an input, to obtain the nonlinear regression model between the input signal representation space and the reference one defined by $S 1$.

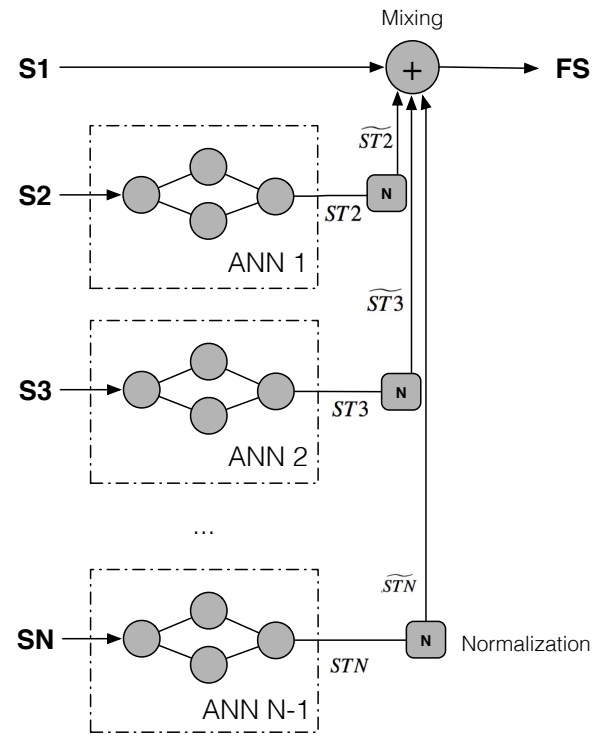

Figure 2: Schema of the distributed fusion model. $S 1$ represents the selected reference signal, $S 2, \ldots, S N$ represent the remaining input signals, $S T 2, \ldots, S T N$ represent the signals generated by the ANNs, $\widetilde{S T 1}, \ldots, \widetilde{S T N}$ are the normalized signals, and $F S$ represents the resulting fused signal.

\subsubsection{Normalization}

The distributed model uses a normalization strategy to each transformed signal $S T 2, S T 3, \ldots, S T N$, obtaining $\widetilde{S T 2}, \widetilde{S T 3}, \ldots, \widetilde{S T N}$ respectively. That 
is,

$$
\widetilde{S T i}(t)=\frac{(S T i(t)-\min (S T i)) \max (S T i * \mathbf{u})}{\max ((S T i-\min (S T i)) * \mathbf{u})}
$$

where $\mathbf{u} \in\{1\}^{30}$ and $*$ denotes the convolution function.

\subsubsection{Weighting and mixing}

Once we have normalized the transformed signals we can linearly mix them together with $S 1$ using the expression

$$
F S(t)=\left(1-\sum_{i=2}^{N} W i(t)\right) S 1(t)+\sum_{i=2}^{N} W i(t) \widetilde{S} i(t)
$$

where $W i(t)$ are weighting the coefficients, defined as

$$
W i(t)=\left\{\begin{aligned}
0 & \text { if } \widetilde{S T i}(t)=0 \wedge|S 1(t)-\widetilde{S T i}(t)|>M+D \\
\frac{1}{N}+\frac{1}{N^{2}+N} & \text { if } S 1(t)=0 \wedge|S 1(t)-\widetilde{S T i}(t)|>M+D \\
1 / N & \text { otherwise }
\end{aligned}\right.
$$

where $M$ is defined as the mean of $|S 1(t)-S T i(t)|, D$ is defined as the standard deviation of $|S 1(t)-S T i(t)|$, and where $N$ is equal to the number of sensors fused. The weighting coefficients $W i(t)$ are defined using the same reasoning as in the case of the centralized model, assigning a priori the same contribution to each signal, but avoiding mixing missed data. Thus the $W i(t)$ coefficient drops the contribution of a signal when the algorithm detects that the values of $S 1$ and $S T i$ differ, and the signal value is zero. The unique differences between the values of the $W i(t)$ weighting coefficients in the centralized and distributed models are due to the differences in the mixing equations of the two approaches (see Equation 2 and Equation 5).

\section{Evaluation methodology}

In this work we have used two data types to test the performance of the two fusion methods presented in the study. The first data type consists of synthetic datasets. These datasets are based on real data and have been simulated by means of generative models based on FDA [51] and MKDE [52] algorithms as described below. In addition, different sensor models have been generated to simulate different responses to the same simulated actigraphy 
signals. In this way we can explore the behavior of the proposed fusion models when varying different characteristics of the sensors, such as noise, sensitivity, percentage of missing data or artifacts.

The second datatype used to test the proposed methods consists of a real dataset acquired during the Help4Mood project, including 5 days of single user activity monitoring using accelerometers embedded in five different devices including wristwatch, belt, keyring, smartphone [53], and undermattress sensor [54]. This second dataset allowed us to test the fusion models in a real scenario.

\subsection{Simulated actigraphy datasets based on real data}

The simulated actigraphy datasets were made by using real data that have been processed and modeled to obtain a generative model of new cases. The steps followed to obtain the set of simulated data are described in the following subsections.

\subsubsection{Real Data used to obtain the generative model}

The real dataset comprises the physical activity of 16 participants monitored 24 hours a day and includes 69 daily actigraphy signals. Half of these participants is composed by controls aimed to follow their normal life routines, while the other half of the participants correspond to patients previously diagnosed with major depression but in the recovered stage at the moment of the study. This dataset was acquired in the framework of Help4Mood EU project [43]. To register the physical activity signals we used the Texas Instruments ez430 Chronos wristwatch as actigraphy sensor using custom algorithms. This watch has a 3-axial accelerometer and each axis was acquired at a sampling frequency of $20 \mathrm{~Hz}$. The algorithm consists on high pass filtering each axis at $1 \mathrm{~Hz}$, computing the time above a threshold (TAT) of $0.1 \mathrm{~g}$ for each axis in epochs of 60 seconds and then the activity index is defined as the maximum TAT over the three axes.

\subsubsection{Missing data detection and imputation}

The detection and imputation of missed data is mandatory before using the real data to build a generative model based on Principal Component Analysis (PCA) descriptors. Missing data is a common problem in actigraphy signals recorded by wearable devices such as wristwatches, smartphones, or key-rings. The main cause of missing data is due to the actigraphy device 
being left unworn, but it could also be due to synchronization errors, empty batteries, or full memory.

In this study a missing data detection algorithm based on the signal filtering and thresholding strategy described in [55] was applied. In the first step the signals were filtered using a moving average filter with a window size equal to $120 \mathrm{~min}$. Once the signal is filtered, it presents a smooth shape and a threshold can be applied to detect regions with very low maintained activity. In this study a threshold equal to 2 TAT was used. Finally a gaussian mean imputation was used to replace missing values.

\subsubsection{Non-linear registration of daily actigraphy signals}

Once the actigraphy signals are free of missing data, it is desirable to perform a non-linear registration of these signals before proceeding to model them. Due to daily routines such as work schedules or sleep-wake cycles, the actigraphy signals contain strong daily patterns. However, although these patterns can be clearly seen in the signal, they do not coincide exactly in time each day, and this phenomenon complicates the analysis and the creation of realistic generative models. To reduce this variability, a non-linear registration technique is applied to align different activity patterns that are slightly phase shifted. In this work the warping algorithm based on FDA library [51] described in [55] was used.

\subsubsection{Feature extraction}

Once we have the actigraphy data preprocessed, and before proceeding with the modeling, we need to describe the information contained in each record of daily activity using a small set of variables. To do so, a standard methodology based on PCA [56] was used. PCA uses an orthogonal transformation, to convert correlated variables into new linearly uncorrelated ones, assuming jointly normally distributed data. In this work the first 15 principal components were used to explain the daily actigraphy data. The number of components was selected attending to the compromise between using a small number of components and conserving most of the original information in the data (in this case $77 \%$ of the variability of the data).

\subsubsection{Modeling and mixture}

As stated, we simulated synthetic data using generative models based on real actigraphy samples. In order to model the real data, we have used a non-parametric strategy based on MKDE [52]. 
MKDE is a nonparametric technique for density estimation that allows us to obtain the probability density function of the features extracted from actigraphy signals. Let $s_{1}, s_{2}, \ldots, s_{r}$ be a set of $r$ actigraphy signals represented as vectors of extracted features (e.g. principal components). Then the kernel density estimate is defined as

$$
\hat{f}_{\mathbf{H}}(s)=\frac{1}{r} \sum_{i=1}^{n} K_{\mathbf{H}}\left(s-s_{i}\right)
$$

where $\hat{f}_{\mathbf{H}}$ is the estimated probability density function, $K$ is the kernel function which is a symmetric multivariate density, and $H$ is the bandwidth (or smoothing) matrix which is symmetric and positive definite. For the generative model presented in this work a MKDE based on a 15-D Gaussian kernel was used. The dimension of the Gaussian kernel corresponds to the 15 principal components used to describe daily actigraphy signals. In the MKDE algorithm, the choice of the bandwidth matrix $H$ is the most important factor size that defines the amount of smoothing induced in the density function estimation. In this study the 1-D search using the max leave-one-out likelihood criterion for automatic bandwidth selection was used.

Using the real data described above and applying the MKDE algorithm we obtained two different probability density functions. The first is based on patient activity records (see Figure 3 top) and the second is based on control activity records (see 3 Figure bottom). In Figure 3 only the first 2 dimensions of the 15 used for signal modeling and simulation could be presented because of the limitations of $2 D$ representations.

\subsubsection{Random samples generation}

Once the generative model is built one can generate random samples using the probability density function obtained with the MKDE algorithm and a random number generation algorithm. For this study we have simulated 20 daily activity patterns using 1 minute of temporal resolution (1440 data-points per pattern). Half of these patterns (10) are simulated using the patient-based probability density function, and the other half (10) are simulated using the control-based probability density function. An example of the random daily actigraphy samples obtained can be seen as green dots in Figure 3. Moreover, an example of a daily actigraphy signal randomly generated from the patient based probability density function is presented in Figure 4 using a black dashed line. 

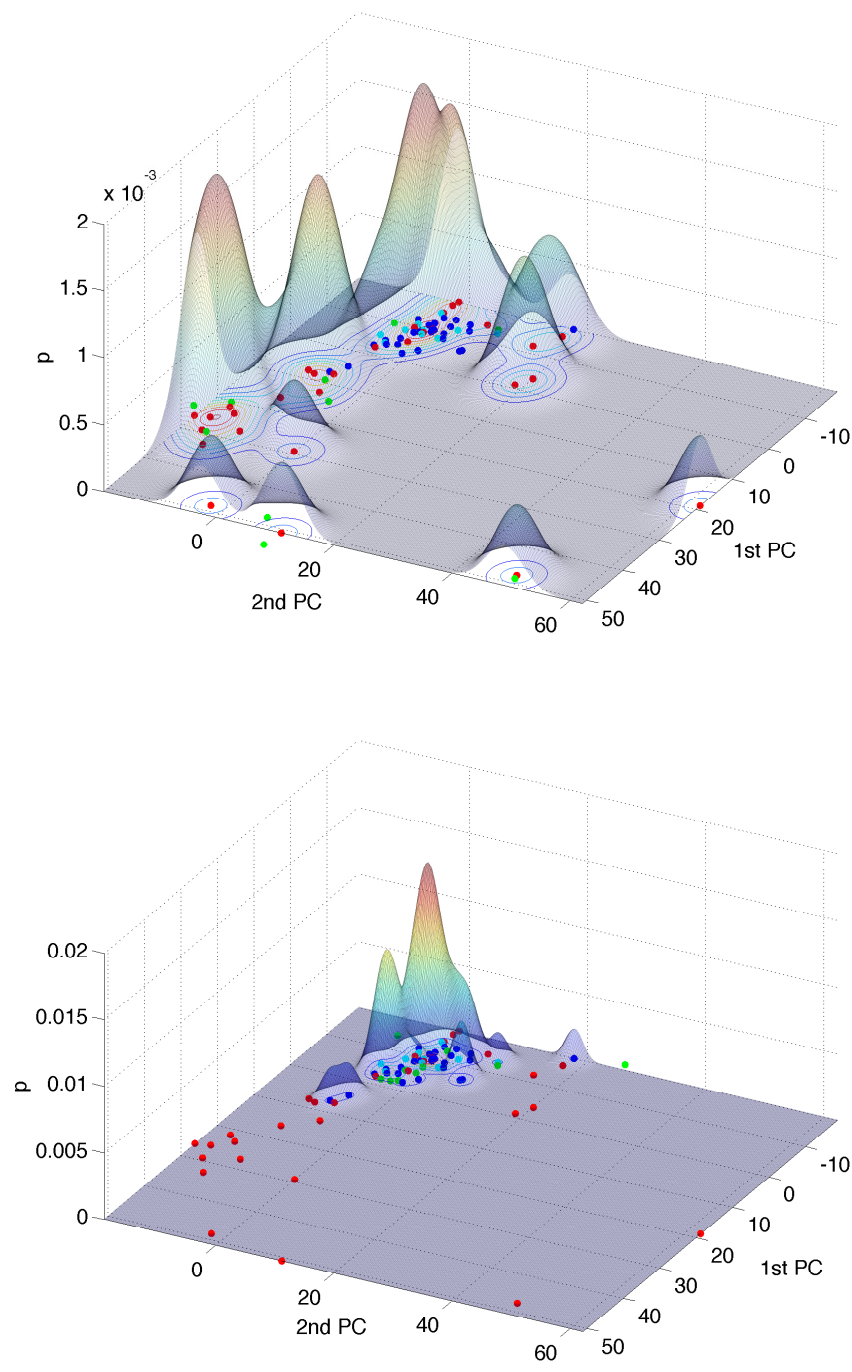

Figure 3: Probability density functions obtained with the MKDE algorithm based on patient activity records (top) and control activity records (bottom). In this figure red dots represent real patient daily actigraphy signals, blue dots represent real control daily actigraphy signals, and green dots represent new daily actigraphy signals generated using the probability density function. 


\subsubsection{Sensor Simulation}

In order to test our fusion methodology we need to have activities registered by different sensors. To achieve this, we consider the randomly generated actigraphy samples as the ideal signals $I S$. We then simulate the degradation of the information generated by each of the sensors. In this work we have considered four main sources of information degradation: noise, low sensitivity, spurious artifacts, and missing data. In addition, different nonlinear responses have been considered to simulate the different response of each sensor to activity.

Noise Regarding noise, uniformly distributed noise controlled by parameter $p_{n}$ has been added to the ideal signal $I S$ as described in the equation below:

$$
S(t)=I S(t)+p_{n} u(t)
$$

where $u(t)$ follows the uniform distribution $\mathcal{U}(0,1), I S$ is the ideal actigraphy signal and $S$ is the transformed signal.

Sensitivity A sensor with low sensitivity is not able to register small activity values. To simulate this effect in the sensor, values below a threshold $p_{s}$ are set to zero,

$$
S(t)=\left\{\begin{aligned}
0 & \text { if } I S(t)<p_{s} \\
I S(t) & \text { otherwise }
\end{aligned}\right.
$$

Artifacts The actigraphy sensors sometimes include activity records not related to the activity of the monitored patient. This can be due to a wide variety of reasons such as an impact over the sensor or errors in the transmission of information. This effect is simulated by the substitution of a piece of the ideal signal by a constant value randomly selected from a uniform distribution $\mathcal{U}$ with values between 0 and 20 . The position of the artifact is also randomly selected using XXXX The width and position of the artifact is defined by the parameter $p_{a}$ as described in the following equations:

$$
S(t)=\left\{\begin{aligned}
\mathcal{U}(0,20) & \text { if } t \in\left[t a_{\text {inf }}, t a_{\text {sup }}\right] \\
I S(t) & \text { otherwise }
\end{aligned}\right.
$$


where $t a_{i n f}$ and $t a_{\text {sup }}$ are defined by

$$
\begin{aligned}
& t a_{\text {inf }}=\mu_{a}-p_{a} \\
& t a_{\text {sup }}=\mu_{a}+p_{a}
\end{aligned}
$$

and the centre of the artifact position $\mu_{a}$ is selected randomly using a uniform distribution $\mu_{a}=\mathcal{U}(0,1441)$

Missing data Missing data are common in actigraphy records. Missing data could be due to various reasons such as synchronization errors, forgetting to wear the actigraph, empty batteries, or full memory. This effect is simulated by the substitution of a piece of the ideal signal by zeros. The width and position of the missed data is defined by the parameter $p_{m}$ as described in the following equations:

$$
S(t)=\left\{\begin{aligned}
0 & \text { if } t \in\left[t l_{\text {inf }}, t l_{\text {sup }}\right] \\
I S(t) & \text { otherwise }
\end{aligned}\right.
$$

where $t l_{\text {inf }}$ and $t l_{\text {sup }}$ are defined by

$$
\begin{aligned}
& t l_{\text {inf }}=\mu_{m}-p_{m} \\
& t l_{\text {sup }}=\mu_{m}+p_{m}
\end{aligned}
$$

and the centre of the missing data position $\mu_{m}$ is selected randomly using a uniform distribution $\mu_{m}=\mathcal{U}(0,1441)$

Non-linear response Each sensor could have a different response depending on the position where the sensor is located on the body (e.g. key ring sensor or wrist watch sensor), or the structural characteristics of the device where the sensor is embedded (e.g. undermattress sensors). To simulate the non-linear response of the actigraphy devices, a second degree function defined by parameters $p_{n l 1}, p_{n l 2}$ and $p_{n l 3}$ was applied to the ideal actigraphy signal. This transformation function is defined in the following equation:

$$
S(t)=p_{n l 1} * I S^{2}(t)+p_{n l 2} * I S(t)+p_{n l 3}
$$


In this study five different sensors have been simulated using the parameters shown in Table 1 and the ideal signals $I S$ obtained by the generative model. The resulting simulated dataset is available at https://www. ibime. webs.upv.es/?p=939. An example of the resulting five simulated sensor signals $(S 1, S 2, S 3, S 4, S 5)$ is presented in Figure 4.

Table 1: Parameters used to generate the signals obtained by the five simulated sensors.

\begin{tabular}{ccccccc} 
parameter & meaning & S1 & S2 & S3 & S4 & S5 \\
\hline$p_{n}$ & noise level & 2 & 3 & 2 & 5 & 2 \\
$p_{s}$ & sensitivity. th. & 2 & 2 & 4 & 4 & 4 \\
$p_{a}$ & artifact width & 3 & 20 & 20 & 20 & 30 \\
$p_{m}$ & miss. data width & 220 & 160 & 320 & 200 & 500 \\
$p_{n l 1}$ & $2^{\text {nd }}$ degree coef. & 4 & 7 & 3 & 3.3 & 2 \\
$p_{n l 2}$ & $1^{\text {st }}$ degree coef. & 3 & 2 & 1 & 5 & 2 \\
$p_{n l 3}$ & const. & 0 & 0 & 0 & 0 & 0
\end{tabular}

\subsection{Real Datasets}

\subsubsection{Data used in the study}

The real dataset used in the study includes a 5 days of single user activity monitoring using accelerometers embedded in five different devices. In addition to the wristwatch we used four more actigraphy devices: one that could be fixed in a belt, one to be used as a keyring, an undermatress sensor, and a smartphone. All the devices, except the smartphone and the undermatress sensor, use the same electronics and algorithms as the wristwatch described on section 3.1.1.

The smartphone was an HTC Wildfire with Android 2.2. The sampling frequency of the embedded three-axial accelerometer is not fix (around 27 $\mathrm{Hz}$ ). For this reason, after the sampling the data was interleaved to obtain a $20 \mathrm{~Hz}$ equivalent frequency. From this point the algorithm is the same as the other actigraphy devices.

The under mattress sensor is also based on the EZ430 Chronos electronics but instead of the accelerometer we used the analog-to-digital converter of 12 bits to acquire two analog signals coming from two different sensors. One of the sensors is sensitive to movements (changes of pressure) and the other detects only the static pressure under the mattress and is used to detect the 


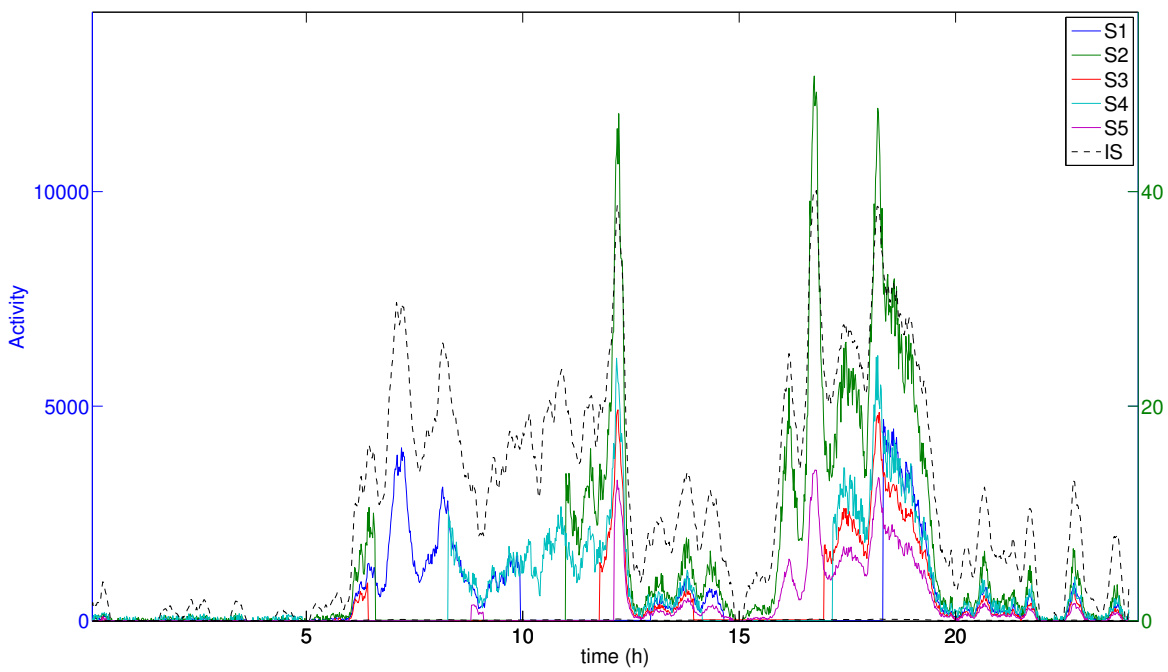

Figure 4: An example of the resulting five sensor signals simulated ( $S 1, S 2, S 3, S 4$ and $S 5$ ) from a ideal signal $I S$ using the parameters in Table 1. Two $y$ axes have been used due to the differences in signal amplitude between $I S$ and the rest of the signals $S 1, S 2$, $S 3, S 4$ and $S 5$. 
presence of a person on the bed. For the movements quantification we also used a time above threshold algorithm with epoch of 60 seconds.

The volunteer was instructed to wear the devices as much as possible during all day and using the undermattress sensor and the watch at night. The wristwatch has to be used in the non-dominant arm, the belt device in a location in the waist and the keyring devices in the usual pocket or pursuit together with the keys. No specific instructions were given for the smartphone. Every night the volunteer has to fulfill a form describing briefly the day activities in periods of 2 hours.

\subsection{Figures of merit}

Two different evaluation strategies have been used in this study. In the case of simulated data, we have used the ideal signals $I S$ as our expected signal. To allow the direct comparison between $I S$ and the fused signals $F S$, we have to transform the fused signals $F S$ into the representation space of $I S$ using the quadratic formula:

$$
\widehat{F S}(t)=\frac{-p_{n l 2} \pm \sqrt{p_{n l 2}^{2}-4 p_{n l 1}\left(p_{n l 3}-F S(t)\right)}}{2 p_{n l 1}}
$$

The transformed signal $\widehat{F S}$ can be obtained easily because we know the non-linear equation used to generate the reference signal $S 1$ (defined by the $p_{n l 1}, p_{n l 2}, p_{n l 3}$ parameters described in Table 1) on which the $F S$ is based.

Once the $\widehat{F S}$ is in the representation space of $I S$ we can compare both signals and obtain an associated error. In this case the error metric used was the Mean Absolute Error (MAE) defined as

$$
M A E=\frac{1}{n} \sum_{t=1}^{n}|\widehat{F S}(t)-I S(t)|
$$

where $n$ is the number of samples in the signal.

In the case of real data, we are not able to use the same strategy because the ideal signal is unknown. To overcome this problem, we have defined our ideal signal $I S$ as the mean of the fused signals obtained using the two fusion methods included in this study when using the five actigraphy signals available.

Finally, another evaluation metric has been used to measure the capability of a fusion method to reduce the effects of missing data or data loss. To do this the evaluation metric is defined as the number of points tagged as missing 
data divided by the total number of points of the signals. The missed data identification algorithm used in this work is described in [55].

\section{Results}

\subsection{Results on simulated datasets}

In this subsection we present the results obtained using the simulated datasets. As has already been mentioned, there are two main advantages of using simulated datasets to evaluate the fusion methods developed. The first is that we can evaluate the error in our fused signals by comparing them to the original signals used for the simulation. This gives us a robust measure of the performance of our methodology. The second advantage is that we are able to test our fusion methods in controlled scenarios, allowing us to perform uniparametric analysis of the influence of each sensor parameter on the performance of the fusion methods.

In order to show the performance of the fusion methods when using simulated datasets we have performed two experiments: The first experiment is focused on the analysis of the influence of input signal characteristics on the performance of fusion algorithms. The second experiment is focused on the analysis of the influence of the number of signals fused on the performance of fusion algorithms. Finally, the result of both fused methods on a small signal sample is presented for illustrative purposes.

\subsubsection{Influence of sensor artifacts on the performance of fusion algorithms}

Using the simulated datasets we are able to study the influence of the most common actigraphy signal problems (missing data, noise, low sensor sensitivity, and artifacts) in the fusion algorithm performance. To do this we have studied the dependence of the MAE of the fused signals on the degradation level of the original simulated signals. In the case of artifacts we have studied the influence of the $p_{a}$ value on the MAE of the fused signals, using different values of $p_{a}$ for all the simulated sensors $\left(p_{a}=[0,30,60,90,120,150,180]\right.$ $\mathrm{min} /$ day). The results can be seen in Figure 5 top. In the case of missing data we have studied the influence of the $p_{m}$ value on the MAE of the fused signals, using different values of $p_{m}$ for all the simulated sensors $\left(p_{m}=[0,133.3,266.7,400,533.3,666.7,800] \mathrm{min} /\right.$ day $)$. The results can be

seen in Figure 5 bottom. In the case of noise we have studied the influence of the $p_{n}$ value on the MAE of the fused signals, using different values of $p_{n}$ for all the simulated sensors $\left(p_{n}=[0,5,10,15,20,25,30]\right)$. The results can 
be seen in Figure 6 top. Finally, in the case of sensitivity we have studied the influence of the $p_{s}$ value on the MAE of the fused signals, using different values of $p_{s}$ for all the simulated sensors $\left(p_{s}=[0,30,60,90,120,150,180]\right)$. The results can be seen in Figure 6 bottom.

\subsubsection{Influence of the number of signals fused on the performance of the fusion algorithms}

The second experiment performed on simulated datasets is designed to evaluate the influence of the number of signals fused on the performance of the fusion algorithms. This experiment highlights the ability of each fusion method to take profit from the additional information added with each input signal included. To achieve this we evaluate two main relevant parameters: the MAE and the percentage of missing data in the fused signal. In this experiment we have used five configurations ranging from a single input signal to five input signals. To obtain the results, all possible combinations of input signals for each configuration have been evaluated and a mean average value has been obtained. That is, for a single input signal we have evaluated 5 combinations, for the two input configuration we have evaluated 4 combinations, for the three input configuration we have evaluated 6 combinations, for the four input configuration we have evaluated 4 combinations, and for the five input configuration the only possible combination. In all cases the signal $S 1$ has been used as a reference signal. Finally, all these experiments have been repeated 10 times to increase the robustness of the results obtained, and the average of these results was computed. The results showing the influence of the number of signals fused on the performance of the fusion algorithms in terms of MAE are presented in Figure 7 top, and the results obtained in terms of percentage of missing data are presented in Figure 7 bottom.

\subsubsection{Example of fused signals}

For illustrative purposes, in Figure 8 we present an example of the fused signals obtained using both fusion methods. This plot includes the fused signals ( $F S$ centralized and $F S$ distributed), the ideal signal $I S$, and the input signals. To help in the comparison between all these signals, all the plotted signals have been transformed to the representation space of the ideal signal using Equation 17.

\subsection{Results on real datasets}

When using real datasets we are not able to change the sensor characteristics to perform uniparametric studies. Therefore the experiments based on 

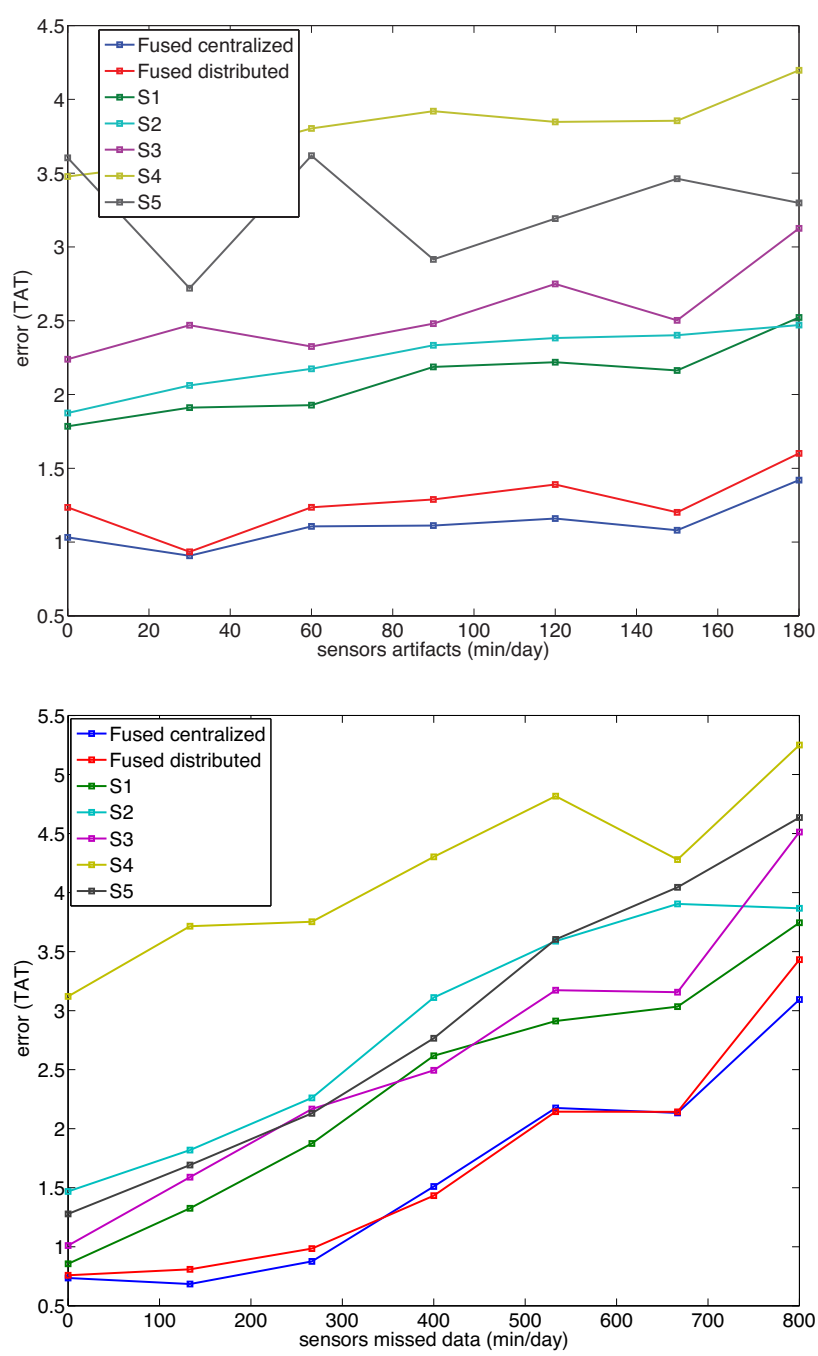

Figure 5: Top: Influence of the sensor artifacts on the MAE of the fused signals obtained using the centralized and distributed approaches (blue and red lines respectively). Bottom: Influence of the amount of missing data on the MAE of the fused signals obtained using the centralized and distributed approaches (blue and red lines respectively). The MAE of the input signals $S 1-S 5$ has been added for comparison purposes. 

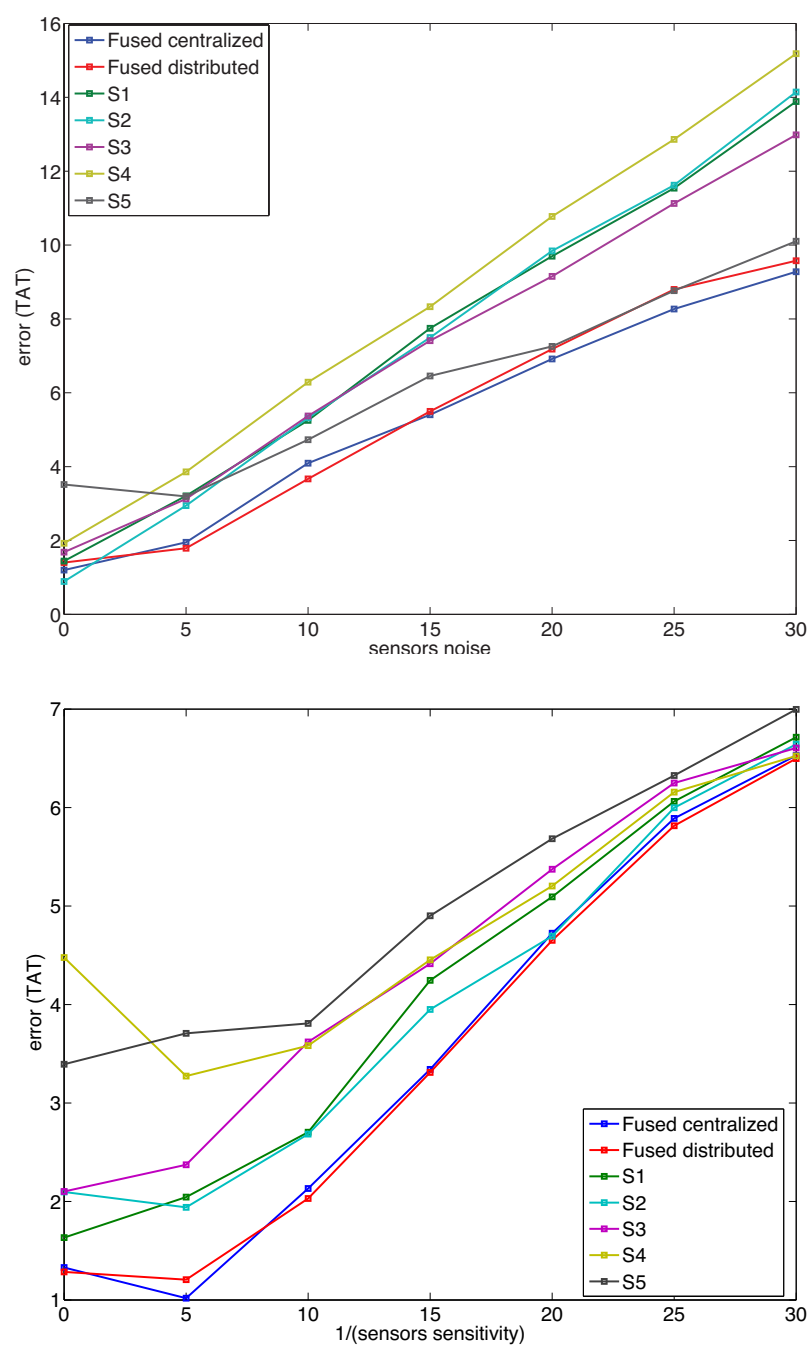

Figure 6: Top: Influence of sensor noise on the MAE of the fused signals obtained using the centralized and distributed approaches (blue and red lines respectively). Influence of the sensor sensitivity on the MAE of the fused signals obtained using the centralized and distributed approaches (blue and red lines respectively). Bottom: The MAE of the input signals $S 1-S 5$ has been added for comparison purposes. 

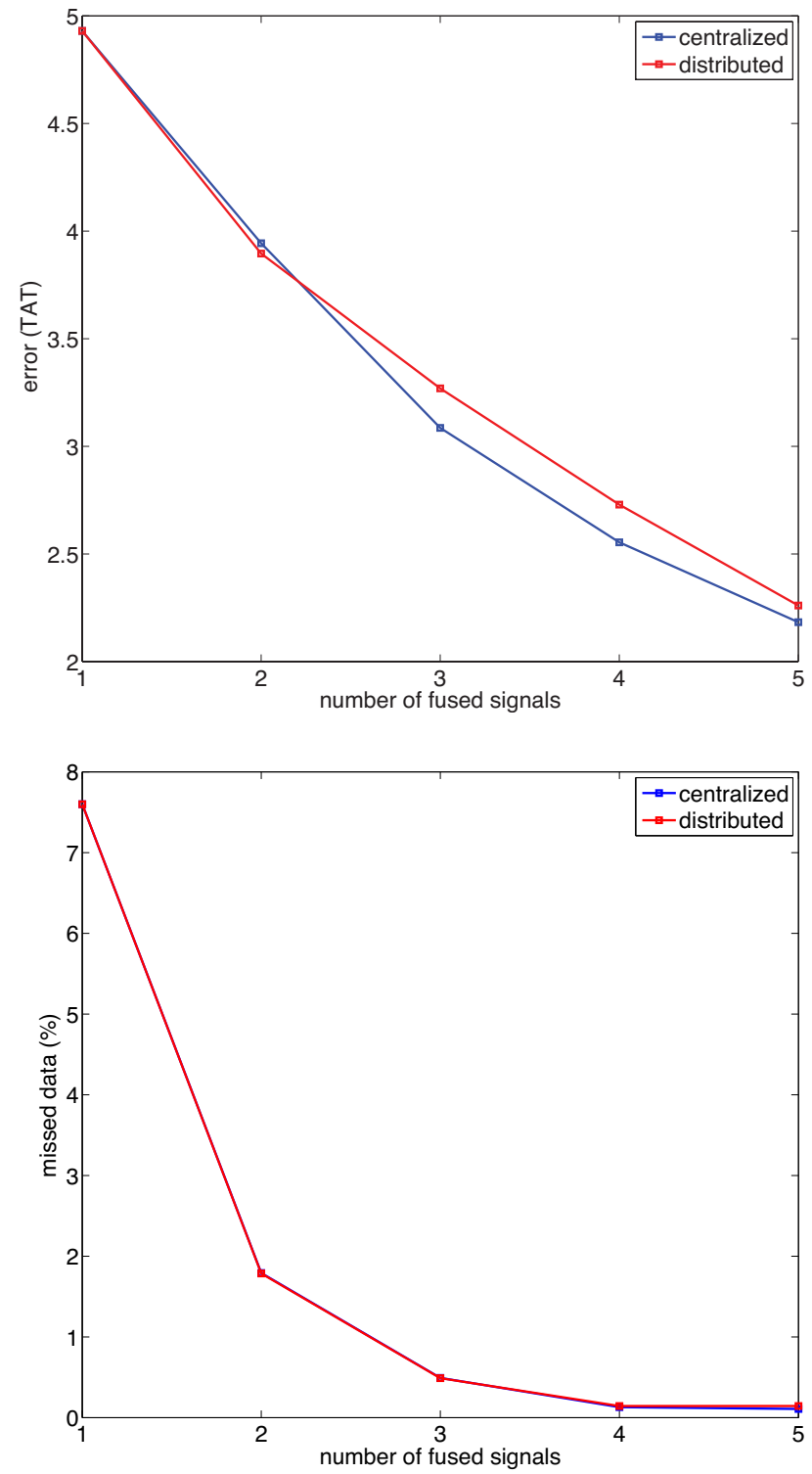

Figure 7: Top: Influence of the number of signals fused on the performance of the fusion algorithms in terms of MAE. Bottom: Influence of the number of signals fused on the performance of the fusion algorithms in terms of percentage of missing data. The results of the centralized fusion method is presented in the blue line while the results of the distributed fusion method is presented in the red line. 


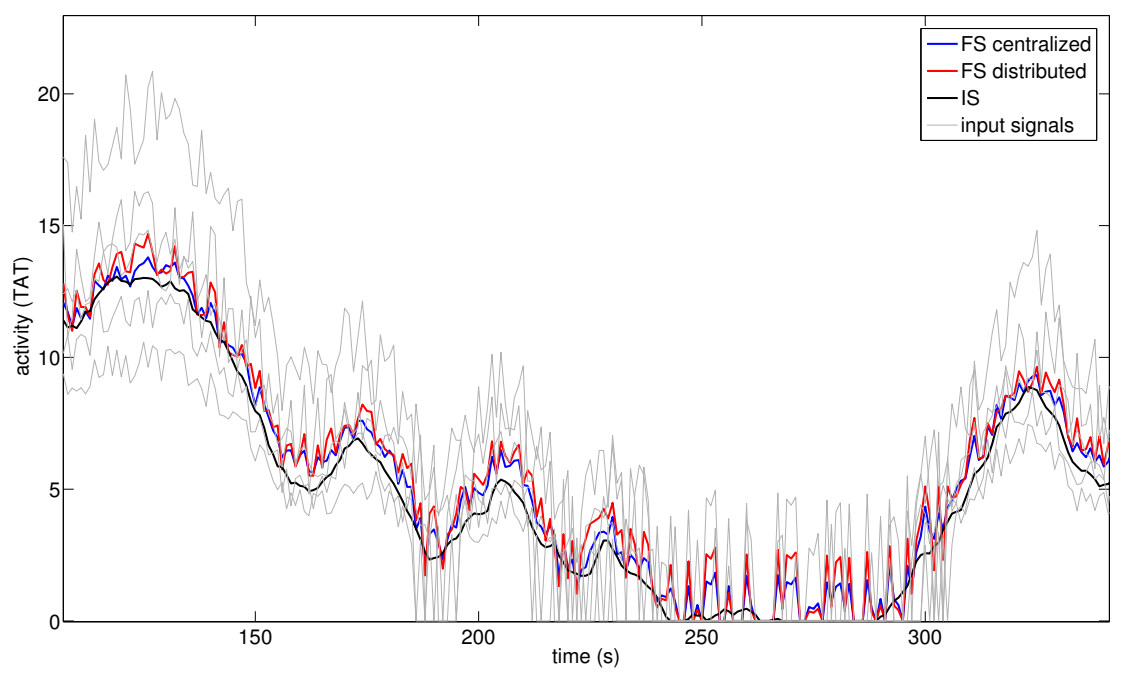

Figure 8: Example of the fused signals obtained using both fusion methods. The fused signal obtained using the centralized method is presented using the blue line, while the distributed one is presented using the red line. Moreover, the ideal signal is presented in the black line, and the input signals are presented in grey lines. To help in the comparison between all these signals, they are transformed to the representation space of the ideal signal $I S$. 
real datasets are limited to the study of the influence of the number of signals fused on the performance of the fusion algorithms. Moreover, in the case of real datasets it is not possible to compare our fused signals with an ideal one because this does not exist. However we can approximate the ideal signal by the signal $\overline{I S}$, obtained as an average of the fused signals $F S$ obtained by both fusion algorithms when using all the input signals available (in this case 5 signals). Using this approach we expect similar behavior to that obtained using simulated datasets.

\subsubsection{Influence of the number of signals fused on the performance of fusion algorithms}

As in the case of the experiment performed using simulated datasets, this experiment is designed to evaluate the influence of the number of signals fused on the performance of the fusion algorithms. The methodology used coincides with the one described for simulated datasets, but in this case we compute the MAE using the approximated $\overline{I S}$ instead of an ideal signal $I S$. The results showing the influence of the number of signals fused on the performance of the fusion algorithms in terms of MAE are presented in Figure 9 bottom, and the results obtained in terms of percentage of missing data are presented in Figure 9 top.

\subsubsection{Example of fused signals}

As in the case of simulated data experiments, we present an example of the fused signals obtained using both fusion methods when using a sample of real data. The plot in Figure 10 includes both fused signals (in the red and blue lines), and the input signals (in the grey lines).

\section{Discussion}

In this work two multi-sensor fusion models for actigraphy signals in the context of outpatient monitoring are presented. Both are based on non-linear regression algorithms based on ANN but using different fusion architectures. The first assumes a centralized paradigm where all the non-reference signals are transformed at the same time and then fused with the reference signal, while the second assumes a distributed paradigm where all the signals are transformed independently, and then fused with the reference signal.

To test the behavior of both models when faced with main actigraphy signal degradation sources, a parametric study was performed based on sim-

ulated datasets. The results of this experiment showed that the fused signals 

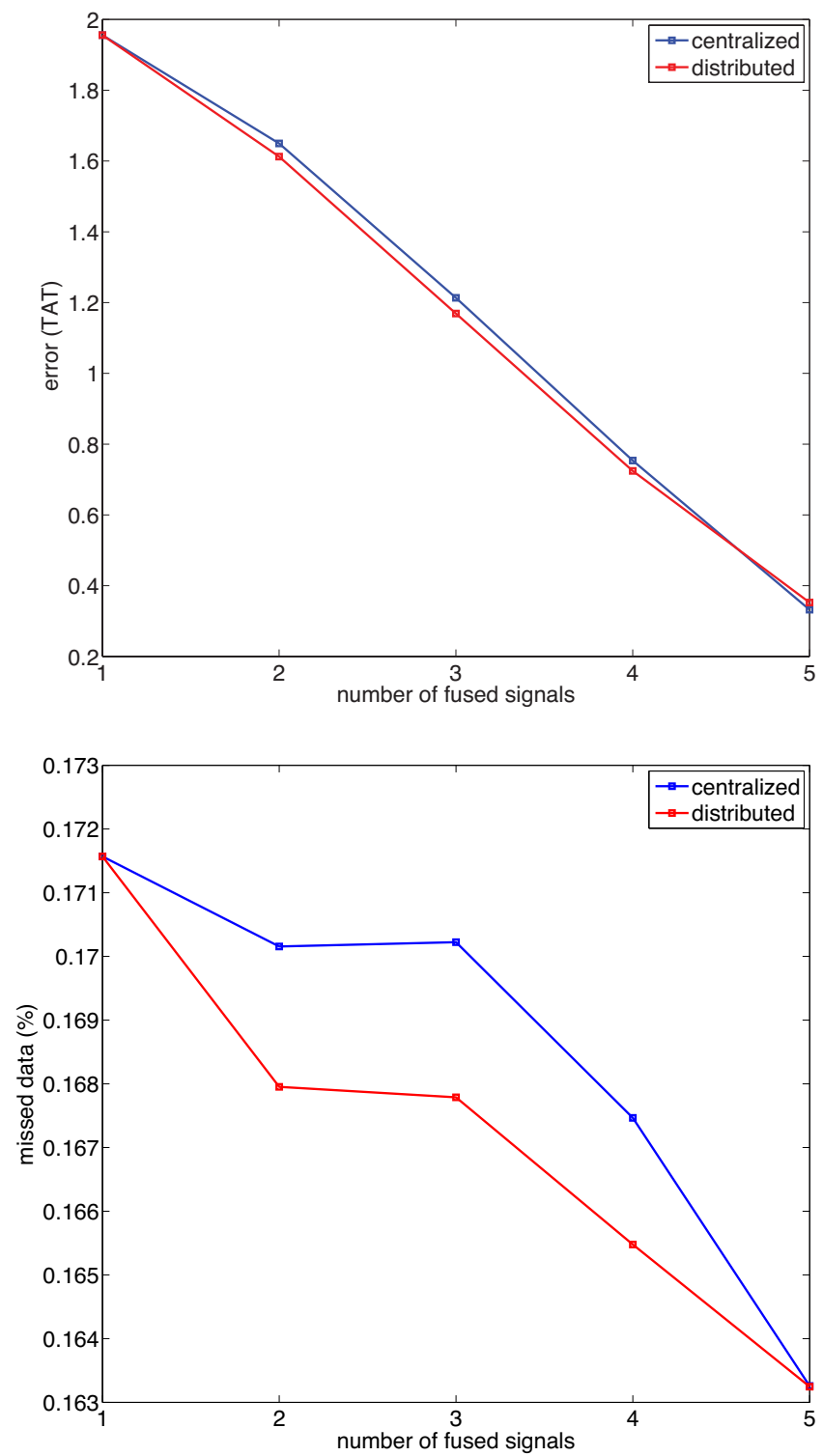

Figure 9: Top: Influence of the number of signals fused on the performance of the fusion algorithms in terms of MAE. Bottom: Influence of the number of signals fused on the performance of the fusion algorithms in terms of percentage of missing data. The results of the centralized fusion method is presented in the blue line while the results of the distributed fusion method is presented in the red line. 


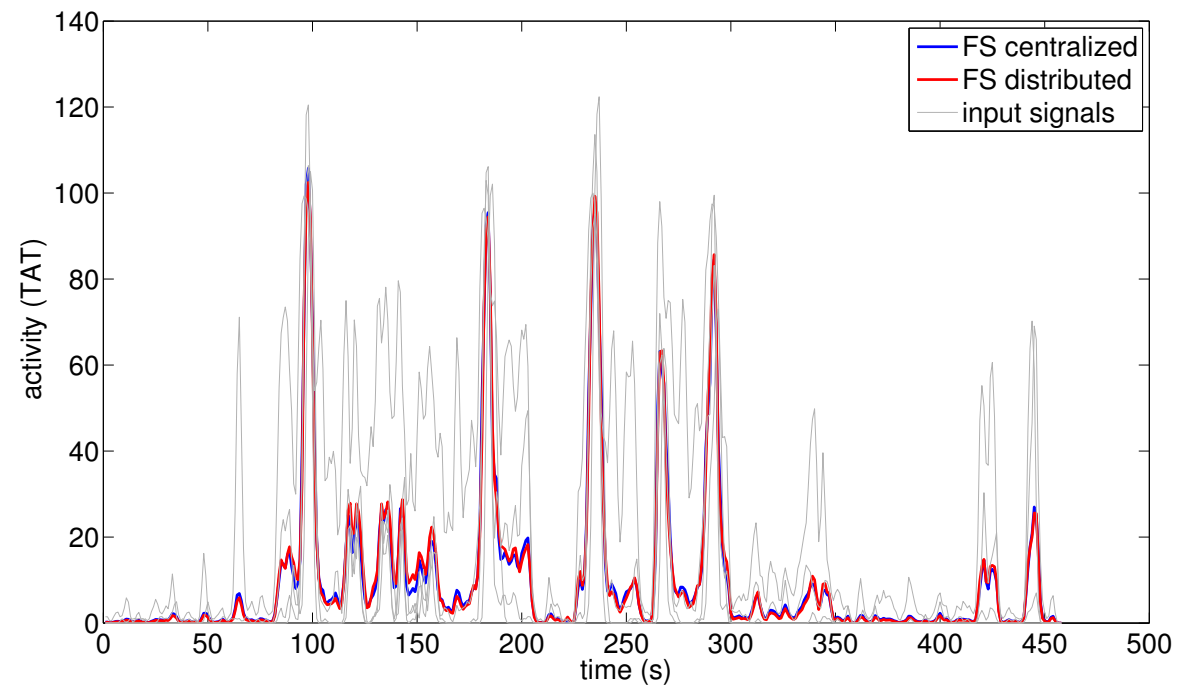

Figure 10: Example of the fused signals obtained using both fusion methods. The fused signal obtained using the centralized method is presented using the blue line, while the distributed one is shown using the red line. The input signals are presented in grey lines. 
obtained using both methodologies improved the results of the original signals in terms of MAE. Using the simulated dataset based on the parameters described on Table 1, the proposed distributed and centralized fusion methods obtained a reduction of $44 \%$ and $46 \%$ respectively of the MAE value of individual input signals (obtained as an average). These results agree with the conclusions obtained by Poh et al. in [57] and in [58], where the authors theoretically analyze the effect of correlation and variance in the fusion of systems with different performances. These studies conclude that if the input signals are different and not correlated, the resulted fused signal will improves the information contained in each of the individual input. In our case study, the input signals are different and not correlated due to data imperfections (such as artifacts, missed data or noise) and differences in sensors characteristics and placement (including different sensor sensitivities). In this sense an improvement of the MAE value was expected.

After the parametric experiment, a study of the influence of the number of signals fused on the performance of the fusion algorithms was performed. This study was carried out using both simulated and real datasets. When using the simulated dataset the results obtained by the proposed multi-sensor fusion models were satisfactory and congruent, obtaining a decrement of the error with the number of input signals added to the fusion algorithms. Analogously, when using real datasets the results show similar behavior to the ones obtained when using simulated datasets. That is, a quasi-linear decrement of the error with the number of input signals added to the fusion algorithms.

In this work we have considered also an analysis of the influence of the number of signals fused on the percentage of missing data. In the experiments performed on simulated datasets the results showed an exponential decrement of the percentage of missing data while increasing the number of input signals, while in the experiments performed on real datasets, the results showed a small linear decrement of the percentage of missing data when increasing the number of input signals.

Finally, and for illustrative purposes, two examples of the application of both fusion methodologies over a simulated data sample and over a real data sample are presented. In the case of simulated data, the fused signals obtained are very similar to the ideal signal even when some of the input signals contain missed data. Analogously, the sample results obtained when using real datasets seem to be congruent with the signal shape expected, although we cannot compare with an ideal signal. 
Comparing both fusion models in the parametric experiment we can see a very similar behavior in terms of performance when the signals are affected by missing data, sensor sensitivity, and noise in all the parameters tested. However in the case of the influence of the sensor artifacts on the MAE of the fused signals, we can see a slightly better behavior of the centralized fusion model across all the parameters tested.

With respect to the influence of the number of signals fused on the performance of the fusion algorithms, we can see also similar behavior in both proposed methods. The results obtained using simulated datasets show that centralized fusion models improve over the results obtained by the distributed fusion model but not significantly. In the case of results based on the real dataset, both fusion models present very similar behavior and MAE values. When analyzing Figures 7 and 9 it is important to notice that the results for one and two fused signals must coincide because the model used in both cases corresponds to the same architecture.

These results are congruent with studies suggesting that a centralized multi-sensor fusion architecture theoretically performs better than a distributed one [59]. However it is important to note that the distributed architecture exhibits many attractive properties such as being scalable in structure without being constrained by centralized computational bottlenecks, or modular in the implementation of fusion nodes [60, 61].

This study focuses on the development of actigraphy fusion models at the level of raw data because this allows us to obtain more accurate actigraphy signals and therefore a more robust analysis of outpatient activity patterns. However, there are other interesting approaches to the fusion of actigraphy information that could addressed in future work. They include the development of actigraphy fusion models at the level of feature fusion or even at the level of fusion of the decision models. That is, to develop fusion models where the inputs are the extracted features of each of the input signals, or the decisions made using the information in each of the signals. These different approaches are described in [4] and will be addressed in future work.

In summary, in this study an exhaustive characterization of two novel raw data fusion models for fusing actigraphy signals in the context of outpatient monitoring have been presented. In the evaluation, both models achieved high performance in terms of robustness to artifacts, missing data, noise, and sensor sensitivity. Moreover, they present a quasi linear decrement of the error with respect to the number of input signals added. We conclude that the models presented here allow reliable monitoring of outpatient physical 
activity by using a set of different devices. As a direct consequence, we expect to facilitate a less intrusive and more dependable way to acquire this clinically valuable information.

\section{Acknowledgements}

This work was partially funded by the European Commission: Help4Mood (contract no. FP7-ICT-2009-4: 248765). E. Fuster-Garcia acknowledges Programa Torres Quevedo from Ministerio de Educación y Ciencia, co-founded by the European Social Fund (PTQ-12-05693).

\section{References}

[1] Y. Hao, R. Foster, Wireless body sensor networks for healthmonitoring applications, Physiological Measurement 29 (11) (2008) R27. doi:10.1088/0967-3334/29/11/R01.

[2] B. Dasarathy, Sensor fusion potential exploitation-innovative architectures and illustrative applications, Proceedings of the IEEE 85 (1) (1997) 24-38. doi:10.1109/5.554206.

[3] R. Luo, C.-C. Yih, K.-L. Su, Multisensor fusion and integration: approaches, applications, and future research directions, IEEE Sensors Journal 2 (2) (2002) 107-119. doi:10.1109/JSEN.2002.1000251.

[4] J. Dong, D. Zhuang, Y. Huang, J. Fu, Advances in multi-sensor data fusion: Algorithms and applications, Sensors (Basel, Switzerland) 9 (10) (2009) 7771-7784. doi:10.3390/s91007771.

[5] B. Khaleghi, A. Khamis, F. O. Karray, S. N. Razavi, Multisensor data fusion: A review of the state-of-the-art, Information Fusion 14 (1) (2013) 28-44. doi:10.1016/j.inffus.2011.08.001.

[6] F. Castanedo, A review of data fusion techniques, The Scientific World Journal 2013 (2013) e704504. doi:10.1155/2013/704504.

[7] H. Durrant-Whyte, T. C. Henderson, Multisensor data fusion, in: B. S. Prof, O. K. Prof (Eds.), Springer Handbook of Robotics, Springer Berlin Heidelberg, 2008, pp. 585-610. 
[8] D. Crisan, A. Doucet, A survey of convergence results on particle filtering methods for practitioners, IEEE Transactions on Signal Processing 50 (3) (2002) 736-746. doi:10.1109/78.984773.

[9] G. Shafer, A Mathematical Theory of Evidence, Princeton University Press, Princeton, N.J., 1976.

[10] B. Bracio, W. Horn, D. Moller, Sensor fusion in biomedical systems, in: Proceedings of the 19th Annual International Conference of the IEEE Engineering in Medicine and Biology Society, 1997, Vol. 3, 1997, pp. 1387-1390 vol.3. doi:10.1109/IEMBS.1997.756639.

[11] A. Benavoli, B. Ristic, A. Farina, M. Oxenham, L. Chisci, An approach to threat assessment based on evidential networks, in: 2007 10th International Conference on Information Fusion, 2007, pp. 1-8. doi:10.1109/ICIF.2007.4408020.

[12] P. J. Escamilla-Ambrosio, N. Mort, A hybrid kalman filter-fuzzy logic architecture for multisensor data fusion, in: Proceedings of the 2001 IEEE International Symposium on Intelligent Control, 2001. (ISIC '01), 2001, pp. 364-369. doi:10.1109/ISIC.2001.971537.

[13] J. Sasiadek, P. Hartana, Sensor data fusion using kalman filter, in: Proceedings of the Third International Conference on Information Fusion, 2000. FUSION 2000, Vol. 2, 2000, pp. WED5/19-WED5/25 vol.2. doi:10.1109/IFIC.2000.859866.

[14] H. Zhu, O. Basir, A novel fuzzy evidential reasoning paradigm for data fusion with applications in image processing, Soft Computing 10 (12) (2006) 1169-1180. doi:10.1007/s00500-005-0039-1.

[15] A.-L. J. Mihai C. Florea, Fusion of imperfect information in the unified framework of random sets theory: Application to target identification, Information Fusion (2007) 142.

[16] H. Borotschnig, L. Paletta, A. Pinz, A comparison of probabilistic, possibilistic and evidence theoretic fusion schemes for active object recognition, Computing 62 (4) (1999) 293-319. doi:10.1007/s006070050026. 
[17] X. Mingge, H. You, H. Xiaodong, S. Feng, Image fusion algorithm using rough sets theory and wavelet analysis, in: 2004 7th International Conference on Signal Processing, 2004. Proceedings. ICSP '04, Vol. 2, 2004, pp. 1041-1044 vol.2. doi:10.1109/ICOSP.2004.1441500.

[18] L. Yong, X. Congfu, P. yunhe, A new approach for data fusion: implement rough set theory in dynamic objects distinguishing and tracing, in: 2004 IEEE International Conference on Systems, Man and Cybernetics, Vol. 4, 2004, pp. 3318-3322 vol.4. doi:10.1109/ICSMC.2004.1400853.

[19] W. Haijun, C. Yimin, Sensor data fusion using rough set for mobile robots system, in: Proceedings of the 2nd IEEE/ASME International Conference on Mechatronic and Embedded Systems and Applications, 2006, pp. 1-5. doi:10.1109/MESA.2006.296962.

[20] O. Basir, F. Karray, H. Zhu, Connectionist-based dempster-shafer evidential reasoning for data fusion, IEEE Transactions on Neural Networks 16 (6) (2005) 1513-1530. doi:10.1109/TNN.2005.853337.

[21] R. Mahler, "Statistics 101" for multisensor, multitarget data fusion, IEEE Aerospace and Electronic Systems Magazine 19 (1) (2004) 53-64. doi:10.1109/MAES.2004.1263231.

[22] R. P. S. Mahler, Statistical Multisource-Multitarget Information Fusion, Artech House, Inc., Norwood, MA, USA, 2007.

[23] S. J. Wellington, J. Atkinson, R. P. Sion, Sensor validation and fusion using the nadaraya-watson statistical estimator, in: Proceedings of the Fifth International Conference on Information Fusion, 2002, Vol. 1, 2002, pp. 321-326 vol.1. doi:10.1109/ICIF.2002.1021169.

[24] P. Ibarguengoytia, L. Sucar, S. Vadera, Real time intelligent sensor validation, IEEE Transactions on Power Systems 16 (4) (2001) 770-775. doi:10.1109/59.962425.

[25] J. Frolik, M. Abdelrahman, P. Kandasamy, A confidence-based approach to the self-validation, fusion and reconstruction of quasi-redundant sensor data, IEEE Transactions on Instrumentation and Measurement 50 (6) (2001) 1761-1769. doi:10.1109/19.982977. 
[26] M. Kumar, D. Garg, R. Zachery, A method for judicious fusion of inconsistent multiple sensor data, IEEE Sensors Journal 7 (5) (2007) 723-733. doi:10.1109/JSEN.2007.894905.

[27] J. He, H. Li, J. Tan, Real-time daily activity classification with wireless sensor networks using hidden markov model, Conference proceedings: ... Annual International Conference of the IEEE Engineering in Medicine and Biology Society. IEEE Engineering in Medicine and Biology Society. Conference 2007 (2007) 3192-3195. doi:10.1109/IEMBS.2007.4353008.

[28] L. Gao, A. K. Bourke, J. Nelson, A system for activity recognition using multi-sensor fusion, Conference proceedings: ... Annual International Conference of the IEEE Engineering in Medicine and Biology Society. IEEE Engineering in Medicine and Biology Society. Conference 2011 (2011) 7869-7872. doi:10.1109/IEMBS.2011.6091939.

[29] L. Gao, A. K. Bourke, J. Nelson, Activity recognition using dynamic multiple sensor fusion in body sensor networks, Conference proceedings: ... Annual International Conference of the IEEE Engineering in Medicine and Biology Society. IEEE Engineering in Medicine and Biology Society. Conference 2012 (2012) 1077-1080. doi:10.1109/EMBC.2012.6346121.

[30] H. Leutheuser, D. Schuldhaus, B. M. Eskofier, Hierarchical, multi-sensor based classification of daily life activities: Comparison with state-of-theart algorithms using a benchmark dataset, PLoS ONE 8 (10) (2013) e75196. doi:10.1371/journal.pone.0075196.

[31] L. Gao, A. K. Bourke, J. Nelson, Evaluation of accelerometer based multi-sensor versus single-sensor activity recognition systems, Medical Engineering \& Physics 36 (6) (2014) 779-785. doi:10.1016/j.medengphy.2014.02.012.

[32] J. J. Guiry, P. van de Ven, J. Nelson, Multi-sensor fusion for enhanced contextual awareness of everyday activities with ubiquitous devices, Sensors (Basel, Switzerland) 14 (3) (2014) 5687-5701. doi:10.3390/s140305687.

[33] M. Shoaib, S. Bosch, O. D. Incel, H. Scholten, P. J. M. Havinga, Fusion of smartphone motion sensors for physical activity recognition, Sensors 14 (6) (2014) 10146-10176. doi:10.3390/s140610146. 
[34] L. Mo, S. Liu, R. X. Gao, D. John, J. W. Staudenmayer, P. S. Freedson, Wireless design of a multisensor system for physical activity monitoring, IEEE transactions on bio-medical engineering 59 (11) (2012) 3230-3237. doi:10.1109/TBME.2012.2208458.

[35] S. Liu, R. X. Gao, D. John, J. W. Staudenmayer, P. S. Freedson, Multisensor data fusion for physical activity assessment, IEEE transactions on bio-medical engineering 59 (3) (2012) 687-696. doi:10.1109/TBME.2011.2178070.

[36] S. B. Patten, J. V. A. Williams, D. H. Lavorato, M. Eliasziw, A longitudinal community study of major depression and physical activity, General hospital psychiatry 31 (6) (2009) 571-575. doi:10.1016/j.genhosppsych.2009.08.001.

[37] L. de Wit, A. van Straten, F. Lamers, P. Cuijpers, B. Penninx, Are sedentary television watching and computer use behaviors associated with anxiety and depressive disorders?, Psychiatry research 186 (2-3) (2011) 239-243. doi:10.1016/j.psychres.2010.07.003.

[38] B. T. Baune, S. Caliskan, D. Todder, Effects of adjunctive antidepressant therapy with quetiapine on clinical outcome, quality of sleep and daytime motor activity in patients with treatment-resistant depression, Human psychopharmacology 22 (1) (2007) 1-9. doi:10.1002/hup.817.

[39] N. Stanley, Actigraphy in human psychopharmacology: a review, Human psychopharmacology 18 (1) (2003) 39-49. doi:10.1002/hup.471.

[40] D. Todder, S. Caliskan, B. T. Baune, Longitudinal changes of daytime and night-time gross motor activity in clinical responders and nonresponders of major depression, The world journal of biological psychiatry: the official journal of the World Federation of Societies of Biological Psychiatry 10 (4) (2009) 276-284. doi:10.3109/15622970701403081.

[41] A. C. Volkers, J. H. M. Tulen, W. W. Van Den Broek, J. A. Bruijn, J. Passchier, L. Pepplinkhuizen, 24-hour motor activity after treatment with imipramine or fluvoxamine in major depressive disorder, European neuropsychopharmacology: the journal of the European College of Neuropsychopharmacology 12 (4) (2002) 273-278. 
[42] A. C. Volkers, J. H. M. Tulen, W. W. van den Broek, J. A. Bruijn, J. Passchier, L. Pepplinkhuizen, Motor activity and autonomic cardiac functioning in major depressive disorder, Journal of affective disorders 76 (1-3) (2003) 23-30.

[43] Help4 Mood Consortium, Help4Mood: a computational distributed system to support the treatment of patients with major depression. URL http://help4mood.info/

[44] Monarca Project, Monitoring, treatment and prediction of bipolar disorder episodes.

URL http://www.monarca-project.eu

[45] ICT4 Depression Consortium, ICT4Depression.

URL http://www.ict4depression.eu

[46] Optimi Consortium, Online predictive tools for intervention in mental illness.

URL /http://www.optimiproject.eu

[47] C. M. Bishop, Neural Networks for Pattern Recognition, Oxford University Press, 1995.

[48] G. Cybenko, Approximation by superpositions of a sigmoidal function, Mathematics of Control, Signals, and Systems 2 (1989) 303-314.

[49] K. G. Sheela, S. N. Deepa, Review on methods to fix number of hidden neurons in neural networks, Mathematical Problems in Engineering 2013. doi: $10.1155 / 2013 / 425740$.

[50] J. Oliver, E. Fuster-Garcia, J. Cabello, S. Tortajada, M. Rafecas, Application of artificial neural network for reducing random coincidences in PET, IEEE Transactions on Nuclear Science 60 (5) (2013) 3399-3409. doi:10.1109/TNS.2013.2274702.

[51] J. O. Ramsay, B. W. Silverman, Functional data analysis, Springer, New York, 2005.

[52] J. S. Simonoff, Smoothing methods in statistics, Springer, New York [etc.], 1996. 
[53] D. Perez-Diaz de Cerio, S. Ruiz Boque, J. Rosell-Ferrer, J. RamosCastro, J. Castro, A wireless sensor network design for the Help4Mood european project, in: European Wireless, 2012. EW. 18th European Wireless Conference, 2012, pp. 1-6.

[54] H. Mahdavi, J. J. Ramos Castro, G. Giovinazzo, M. Á. García González, F. J. Rosell Ferrer, A wireless under-mattress sensor system for sleep monitoring in people with major depression, in: IASTED International Conference Biomedical Engineering (BioMed). "Proceedings of the ninth IASTED International Conference Biomedical Engineering (BioMed 2012)", 2012, pp. 1-5.

[55] E. Fuster-Garcia, J. Juan-Albarracín, A. Bresó, J. M. Garca-Gómez, Monitoring changes in daily actigraphy patterns of free-living patients, in: International Work-Conference on Bioinformatics and Biomedical Engineering (IWBBIO) proceedings., 2013, pp. 685-693.

[56] I. T. Jolliffe, Principal component analysis, Springer, New York, 2002.

[57] N. Poh, S. Bengio, Why do multi-stream, multi-band and multi-modal approaches work on biometric user authentication tasks?, in: IEEE Int'l Conf. Acoustics, Speech, and Signal Processing (ICASSP), Vol. 5, 2004, pp. 893-896.

[58] N. Poh, S. Bengio, How do correlation and variance of base-experts affect fusion in biometric authentication tasks?, IEEE Transactions on Signal Processing 53 (11) (2005) 4384-4396. doi:10.1109/TSP.2005.857006.

[59] L. Y. Pao, Distributed multisensor fusion, in: GNC, 1994, pp. 1-10.

[60] S. Grime, H. F. Durrant-Whyte, Data fusion in decentralized sensor networks, Control Engineering Practice 2 (5) (1994) 849-863. doi:10.1016/0967-0661(94)90349-2.

[61] N. Xiong, P. Svensson, Multi-sensor management for information fusion: issues and approaches, Information Fusion 3 (2) (2002) 163-186. doi:10.1016/S1566-2535(02)00055-6. 